\title{
Adaptive loading ball traction drive reducer: modeling and efficiency performance
}

\author{
Qing LUO*, Guang-jian WANG*,**, Yu-jiang JIANG* and Shuai-dong ZOU* \\ * State Key Laboratory of Mechanical Transmissions, Chongqing University \\ 174 Shazheng Street, Shapingba District, Chongqing 400044, China \\ E-mail: gjwang@cqu.edu.cn \\ ** Chongqing College of Electronic Engineering \\ 76 East University City Road, Shapingba District, Chongqing, 400044, China
}

Received: 29 January 2021; Revised: 23 March 2021; Accepted: 8 June 2021

\begin{abstract}
In this paper, a novel type of adaptive loading ball traction drive reducer (AL-BTDR) is proposed and investigated in modeling and efficiency performance. A combination of power flow and equal linear velocity method is developed to evaluate the speed ratio for the first time. Meanwhile, the spin loss at contact areas is also analyzed based on the Hertz contact theory, and a new approach depended on power flow is introduced to establish the efficiency model. By applying the efficiency model, the performance of efficiency with different output torque is calculated, and the distinctions under adaptive loading and constant loading are investigated. The results show that AL-BTDR has an excellent efficiency performance under adaptive loading with a maximum of $91 \%$. Additionally, efficiency is higher under adaptive loading and will not sharply drop when compared with constant loading.
\end{abstract}

Keywords : Traction drive, Power flow, Spin loss, Adaptive loading, Efficiency

\section{Introduction}

Recent developments in the field of intelligent manufacturing have led to a renewed interest in robot joint drive systems that set a high requirement for the reducers in miniaturization, efficiency, and transmission accuracy. For meeting the requirements, researchers have put forward many ideas, among which harmonic reducer and cycloid reducer have been applied in the robot drive system (Mahanto et al., 2018; Qian et al., 2020). At the same time, several new motors have been made and used in engineering practice (Chen et al., 2018; Matsuda et al., 2017), which brings new opportunities and challenges for the reducers industry. However, little research has been carried out about this. Thus, a reducer, potential superior in high speed, efficiency, high speed ratio, miniaturization, and transmission accuracy, is such a pressing need and will be the focus of the reducer industry in the future.

In the world of drive systems, some factors limit the application scope of harmonic and cycloid reducers such as lowspeed characteristic, temperature rise, vibration loss, and efficiency performance (Georgiev and Burdick, 2017), although they have advantages in output torque capacity and speed ratio. Comparing to gear drive systems, the high contact pressure and low capacity of the traction drives make it hard to be applied in low speed and heavy load situations. However, the traction drives have an inherent advantage in high speed, miniaturization, efficiency performance, transmission accuracy, and low vibration. Therefore, considerable attention from researchers has been paid to traction drives that have been accelerated by a deeper understanding of rolling contact, new synthetic traction lubricants, and the emergence of materials with high contact capability (Cretu and Glovnea, 2003; Flugrad and Qamhiyah, 2004; Loewenthal et al., 1981).

In general, the traction drives perform as the way: a layer of high-pressure lubricating film has been formed between the traction parts under the action of contact pressure, then, the power and motion are transmitted when relative sliding occurs. At the same time, it can reduce the wear and vibration by separating the traction parts from each other and avoiding contact directly (Ai et al., 2005; Baejin et al., 2004; Sharif et al., 2004). Due to these benefits, the Nasvytis 
traction drive (Loewenthal et al., 1981), self-actuating traction drive reducer (Flugrad and Qamhiyah, 2004), and kinds of CVTs (including belt CVT (Bertini et al., 2014; Jean-Philippe and Philippe, 2010; Okuda et al., 2017), toroidal CVT (Miyata and Liu, 2007; Tyreas and Nikolakopoulos, 2016), and ball CVT (Akehurst et al., 2006)) have been applied in industrial applications.

Although the power loss of traction drives diverges from different types, studies over the past have proved that the power loss is comprised of speed loss and torque loss that are respectively determined by spin and slip (creep) (Ghariblu et al., 2014; Kong et al., 2018; Zhang et al., 1999). Furthermore, lots of literature agree that spin is the primary cause of power loss and efficiency loss (Akehurst et al., 2005; Flugrad and Qamhiyah, 2004; Li et al., 2017; Tomaselli et al., 2019; Tomaselli et al., 2020). Akehurst (Akehurst et al., 2005) figured out that spin would generate shear stress (different from the expected traction direction) in the lubricant film, resulting in power loss and the local temperature rising. Based on creep theory, Hirofumi Itagaki (Itagaki et al., 2020) proposed a modified method to estimate traction curves and found that the traction drive coefficient would reduce with spin increasing. Li X. M (Jiang et al., 2008; Li et al., 2010) analyzed the elastohydrodynamic lubrication contact with spin considered. Their research demonstrated that the minimum oil film thickness would decrease by the increase of spin, and the symmetry of the film shape got lost.

Much work has been done to limit or eliminate the spin loss for achieving an excellent efficiency performance. Yamamoto (Yamamoto et al., 2001) established the efficiency model of half toroidal CVT and pointed out that the spin loss could be decreased by reducing the contact angle. Based on two zero-spin types, Li Qingtao (Li et al., 2015a) came up with a logarithmic disc generatrix that eliminates spin loss theoretically. Besides, researchers have also expended their effort on efficiency modeling that plays a critical role to evaluate the traction drive. Verbelen (Verbelen et al., 2018) contrasted the models of full and half toroidal CVTs in terms of ratio variation and efficiency performance. The result of their work showed that half toroidal CVT was more superior to the full one. Afrabandpey (Afrabandpey and Ghariblu, 2018) studied the contact performance between traction parts to evaluate the efficiency of ball CVT and half toroidal CVT. Tomaselli (Tomaselli et al., 2019; Tomaselli et al., 2020) established the efficiency model of NuVinci with slip and spin considered to evaluate the performance of single-idler and double-idler geometries. They found that the efficiency of single-idler is superior to the double-idler over the entire range of speed ratio.

In consideration of the transmission development trend of the robot joint drive systems, an adaptive loading ball type traction reducer is proposed for the first time in this paper, which is composed of a V-type loading cam and a closed differential planetary mechanism. The new type structure makes it more preponderant in speed ratio (about 35) and dimension than the current fixed axis type traction drives (usually with a lower speed ratio). Besides, combined with the low vibration and excellent efficiency characteristics of traction drives, it is possible for the adaptive loading ball traction reducer to work with new type motors for the low vibration and excellent efficiency characteristics. At the same time, some new methods have been introduced to analyze the performance of the adaptive loading ball traction reducer in terms of modeling and efficiency. Section 2 deduces the models of the ideal and actual speed ratio. The mathematical models of efficiency are established based on the power flow direction in section 3 . The efficiency performance at different output torque is calculated and discussed in section 4 , and the distinctions of efficiency under adaptive and constant loading are also investigated.

$$
\begin{array}{ll}
\text { Nomenclature } & \\
R_{\text {in }, i} & \text { inner radius } i,(i=1,2) ;[\mathrm{mm}] \\
R_{\text {out }, i} & \text { outer ring radius } i,(i=1,2) ;[\mathrm{mm}] \\
R_{\text {ind }, i} & \text { radius of inner curvature to datum } i,(i=1,2) ;[\mathrm{mm}] \\
R_{\text {outd }, i} & \text { radius of outer ring curvature center to datum } i,(i=1,2) ;[\mathrm{mm}] \\
R_{\text {planet }, i} & \text { planet roller radius } i,(i=1,2) ;[\mathrm{mm}] \\
R_{\text {ideler }, i} & \text { idler radius } i,(i=1,2) ;[\mathrm{mm}] \\
R_{\text {orbit }, i} & \text { planetary orbit radius } i,(i=1,2) ;[\mathrm{mm}] \\
R_{\text {idler }} & \text { radius of idler center to datum; }[\mathrm{mm}]
\end{array}
$$




\begin{tabular}{|c|c|}
\hline$\mu$ & traction coefficient; [-] \\
\hline$\delta$ & the angle of groove depth; $\left[^{\circ}\right]$ \\
\hline$\alpha$ & the outer ring contact angle of the first stage; $\left[{ }^{\circ}\right]$ \\
\hline$\beta$ & the outer ring contact angle of the second stage; $\left[{ }^{\circ}\right]$ \\
\hline$\xi_{i}$ & the angle of planetary orbit radius and the line from roller to idler $i,(i=1,2) ;\left[^{\circ}\right]$ \\
\hline$\tau_{i}$ & the angle of idler center to datum and the line from roller to idler $i,(i=1,2) ;\left[{ }^{\circ}\right]$ \\
\hline$\omega_{i}$ & ideal angular velocity $i,(i=1-8) ;[\mathrm{rad} / \mathrm{s}]$ \\
\hline $\boldsymbol{\omega}_{\text {planet }, i}$ & ideal self-rotating angular velocity of planetary roller $i,(i=1,2) ;[\mathrm{rad} / \mathrm{s}]$ \\
\hline$\omega_{\text {orbit }}$ & ideal angular velocity of planetary orbit; $[\mathrm{rad} / \mathrm{s}]$ \\
\hline$\omega_{i}^{\prime}$ & actual angular velocity $i,(i=1-8) ;[\mathrm{rad} / \mathrm{s}]$ \\
\hline$\omega_{\text {planet }, i}^{\prime}$ & actual self-rotating angular velocity of planetary roller $i,(i=1,2) ;[\mathrm{rad} / \mathrm{s}]$ \\
\hline$\omega_{\text {orbit }}^{\prime}$ & actual angular velocity of planetary orbit; $[\mathrm{rad} / \mathrm{s}]$ \\
\hline$\omega_{\text {spin }, i}$ & actual spin angular velocity of contact area $i,(i=1-4) ;[\mathrm{rad} / \mathrm{s}]$ \\
\hline $\boldsymbol{T}_{i}$ & torque of component $i,\left(i=1-8, \mathrm{H}_{1}, \mathrm{H}_{2}\right) ;[\mathrm{Nm}]$ \\
\hline$c_{r, i}$ & creep of contact area $i,(i=1-4) ;[-]$ \\
\hline$C_{r}$ & global creep;[-] \\
\hline$n_{l}$ & number of loading roller; [-] \\
\hline$n_{p, i}$ & number of planet roller $i,(i=1,2) ;[-]$ \\
\hline$\eta_{i}$ & efficiency of contact area $i,(i=1-4) ;[-]$ \\
\hline$\eta$ & overall efficiency;[-] \\
\hline
\end{tabular}

\section{Traction drive model}

\subsection{Geometrical description}

The model of adaptive loading ball type traction drive reducer is shown in Fig. 1(a). It is surprising to note that the structure is similar to Motion CVT (Jeremy and Don, 2003) that has been proved with high-efficiency performance. At the same time, Fig. 1(b) shows the positional relationship between the planet rollers and the idlers. Each stage planetary unit (corresponded to $\mathrm{X}$ and $\mathrm{Y}$ in Fig. 1(c)) includes the following main parts: an inner ring; two separated outer rings; a number $n_{p, i}(i=1,2)$ of spherical rollers and idlers. Moreover, the adaptive loading cam involves a loading plate, a number $n_{l}$ of loading rollers, and a V-shaped groove.

Under the steady-state, the two inner rings rotate at the same speed as the input shaft, then, the planetary rollers of the first stage will drive the idlers and planetary carrier. Because of the idlers of second stage connected with the one of first stage by planetary carriers, the second stage unit becomes a closed differential transmission system that has an edge in speed ratio and dimension. Moreover, it has the potential to further reduce the size by integrating the planet balls and idlers and eliminating the idler bearings, which can weaken the concept of planetary carrier in some extent (similar to the structure in (Brassitos and Jalili, 2017)). When the external load changes, a slight angular displacement occurs in the outer ring of the first stage, and the force acting on the loading rollers and outer ring will alter through the groove, which enables the proposed one to realize adaptive loading. 


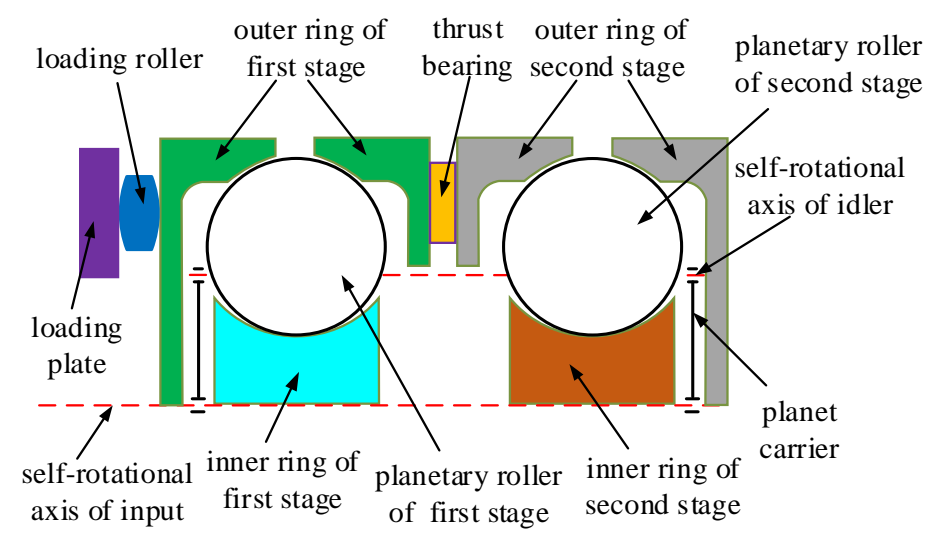

(a)

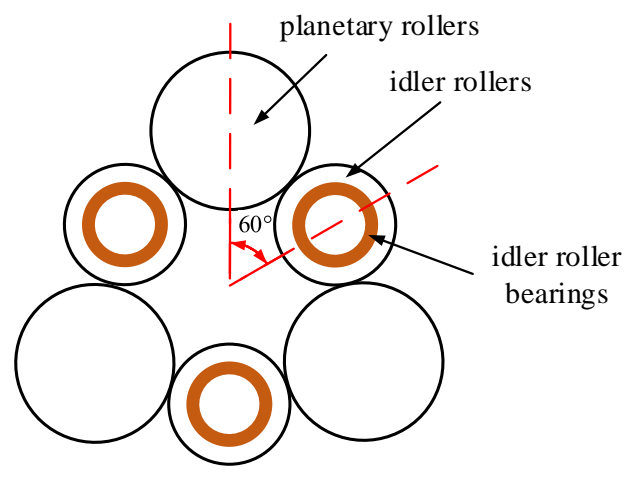

(b)

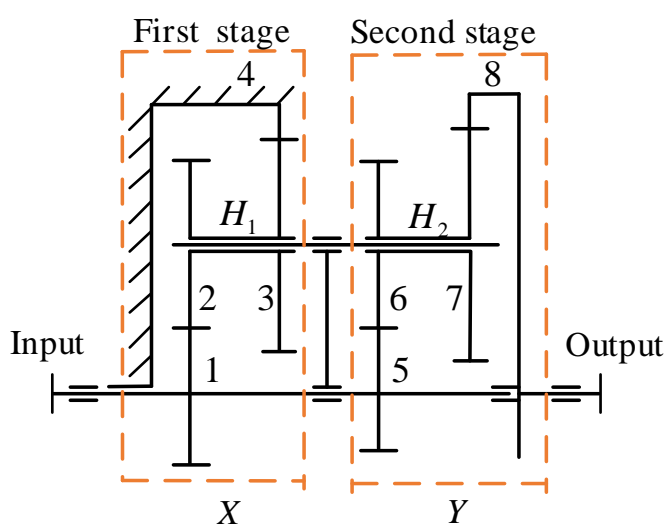

(c)

1-the first stage sun gear; 2-the first stage planet gear 1; 3-the first stage planet gear 2; 4-the first fixed ring; 5- the second stage sun gear; 6-the second stage planet gear 1; 7-the second stage planet gear 2; 8-the output ring; $\mathrm{H}_{1} / \mathrm{H}_{2}$-the planet carrier; Fig. 1. (a) The structural model of AL-BTDR (b) The relations between planet rolls and idlers (c) Equivalent transmission schematic of AL-BTDR

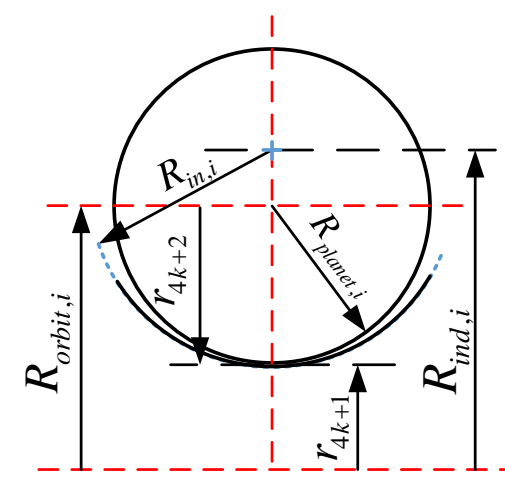

(a)

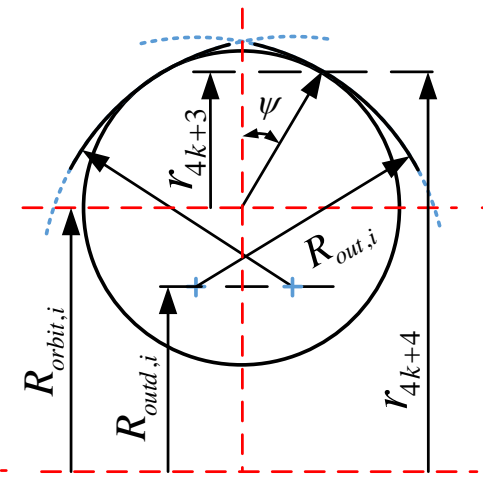

(b)

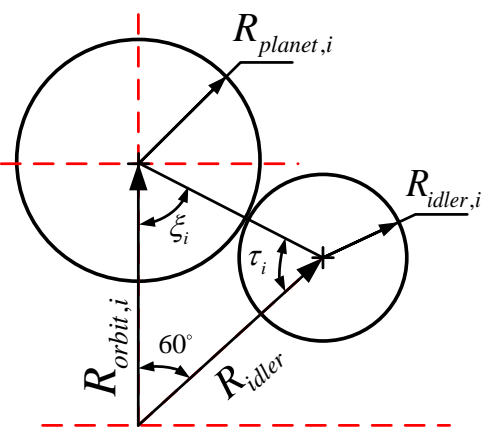

(c)

Fig. 2 Geometry of traction parts: (a) Inner raceways and planet rollers (b) Outer raceways and planet rollers (c) Idlers and planet rollers

Figure 2 presents the geometric correlations between the planetary rollers and the inner raceways, outer raceways, and idlers, where $\psi$ is the contact angle between the outer ring and planetary roller ( $\alpha$ for the first stage, $\beta$ for the second stage), $r_{4 k+i}(k=0,1 ; i=1,2,3,4)$ represents the equivalent contact radius of transmission components. According to Fig. 2, the geometric model can be expressed by Eq. (1) and Eq. (2). 


$$
\begin{aligned}
& \left\{\begin{array} { l } 
{ r _ { 1 } = R _ { \text { ind } , 1 } - R _ { \text { in } , 1 } } \\
{ r _ { 2 } = R _ { \text { planet } , 1 } \operatorname { c o s } \alpha } \\
{ r _ { 3 } = R _ { \text { planet } , 1 } - R _ { \text { out } , 1 } \operatorname { c o s } \alpha } \\
{ r _ { 4 } = R _ { \text { outd } , 1 } }
\end{array} \quad \left\{\begin{array}{l}
r_{5}=R_{\text {ind }, 2}-R_{\text {in }, 2} \\
r_{6}=R_{\text {planet }, 2} \cos \beta \\
r_{7}=R_{\text {planet }, 2} \cos \beta \\
r_{8}=R_{\text {outd }, 2}-R_{\text {out }, 2} \cos \beta
\end{array}\right.\right. \\
& R_{\text {orbit }, 1}=r_{1}+r_{2} \quad R_{\text {orbit }, 2}=r_{5}+r_{6}
\end{aligned}
$$

\subsection{Kinematics description}

Thus far, a number of studies have explored the kinematics of traction drive. Akehurst (Akehurst et al., 2005) pointed out that there must be a specific slip at the ellipse contact area for generating shear stress between the lubricant film during transmitting, and the slip could be divided into three different components: relative longitudinal movement (slip), relative motion perpendicular to the longitudinal direction (slide slip), relative rotational motion around the axis perpendicular to the contact area (spin). The work also indicated that spin loss was the primary loss than the slip loss, and the slide slip loss could be negligible in the whole transmission ratio range. Hence, the following assumptions are made for simplification.

1) The reducer is well-assembled and pre-tightened;

2) Hertz elastic deformation occurs at the ellipse contact areas;

3) Overlook the impact of slide slip during transmission;

4) Ignoring the influence of temperature and viscous pressure on the traction medium.

\subsubsection{Ideal speed ratio}

As can be seen from Fig. 3, a graphic analysis approach based on equal linear velocity is adopted to dissect the ideal speed ratio with the stipulation that the positive direction of the linear velocity coincides with the rotating direction of the inner ring, and the circle or arc in Fig. 3 corresponds to the transmission parts. Referring to the stipulation, $O_{1}$ and $\mathrm{O}_{2}$ are the absolute instant centers, and $V_{H_{1}}$ as well as $V_{H_{2}}$ indicate the linear velocity at the center of the planet carrier. From the graph, we can see that the angular velocity is a tangent function of angle that can be obtained through the triangle composed by the radius and linear velocity vector of transmission parts. (For example, the angular velocity of component 1 can be described by the tangent of $\left.\angle A_{1} O_{1} B_{1}\right)$.

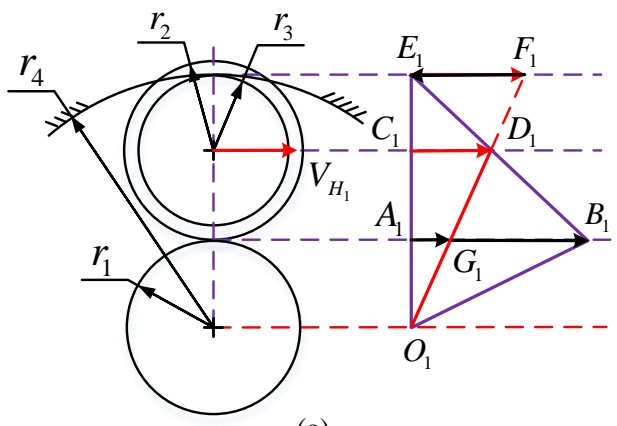

(a)

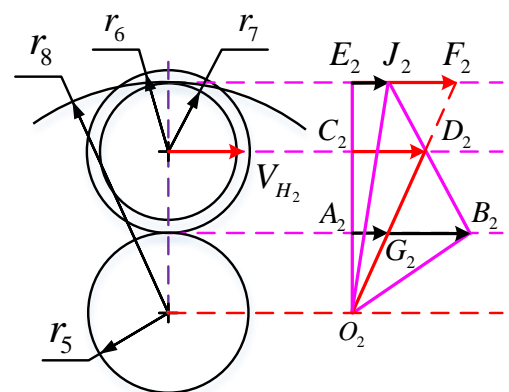

(b)

Fig. 3 (a) Ideal angular velocity of the first stage

(b) Ideal angular velocity of the second stage

Then, the ideal speed ratio can be respectively stated as Eq. (3) and Eq. (4) in the light of Fig. 3, where $i_{1 H_{1}}$ is the ideal speed ratio of $\mathrm{X}$, and $i_{18}$ is the total ideal speed ratio.

$$
\begin{aligned}
& i_{1 H_{1}}=\frac{\omega_{1}}{\omega_{\text {orbit }}}=\frac{\overrightarrow{A_{1} B_{1}} / O_{1} A_{1}}{\overline{C_{1} D_{1}} / O_{1} C_{1}}=1+\frac{r_{2} r_{4}}{r_{1} r_{3}} \\
& i_{18}=\frac{\omega_{1}}{\omega_{8}}=\frac{\omega_{5}}{\omega_{8}}=\frac{\overrightarrow{A_{2} B_{2}} / O_{2} A_{2}}{\overline{E_{2} J_{2}} / O_{2} E_{2}}=\frac{1}{\frac{1}{i_{1 H 1}}-\frac{r_{5} r_{7}}{r_{6} r_{8}}\left(1-\frac{1}{i_{1 H 1}}\right)}
\end{aligned}
$$




\subsubsection{Actual speed ratio}

In the case of the actual operation process, perfect rolling is not feasible since the creep is inevitable, resulting in inequality between the ideal and actual speed ratio. Although some researches have been carried out on the actual speed ratio of traction drives, there have been few investigations into the planetary transmission system because of restricting to the axis-fixed transmission systems. Therefore, a novel algorithm founded on power flow and equal linear velocity is developed here to estimate the actual speed ratio and global creep. The algorithm comprises the following four steps:

I) Determining the power flow direction of traction components without power loss;

II) Adjudging the driving-driven relationship of traction components without power loss in the conversion mechanism;

III) Defining the creep at contact areas in the conversion mechanism;

IV) Modeling and estimating the actual speed ratio and global creep.

I) Determining the power flow direction of traction components without power loss

Combined with (Chen, 2013; Chen and Jorge, 2006), Fig. 4 illustrates the simplified model of traction components without power loss, where the graph line, the hollow circle, and the boxes individually represent the traction drive parts, power nodes, and traction drive systems ( $\mathrm{X}$ and $\mathrm{Y}$ ) with the provision that the power flowing into the traction drive system is positive and the one flowing out is negative.

The following equations are acquired without efforts based on the balance of torque and power.

$$
\left\{\begin{array}{l}
\boldsymbol{T}_{0}+\boldsymbol{T}_{8}+\boldsymbol{T}_{4}=0 \\
\boldsymbol{T}_{0} \cdot \boldsymbol{\omega}_{0}+\boldsymbol{T}_{8} \cdot \boldsymbol{\omega}_{8}+\boldsymbol{T}_{4} \cdot \boldsymbol{\omega}_{4}=0
\end{array}\right.
$$

Referring to Eq. (5), it can be found that:

$$
\boldsymbol{T}_{0}: \boldsymbol{T}_{8}: \boldsymbol{T}_{4}=1:-i_{18}^{4}:\left(i_{18}^{4}-1\right)
$$

Similarly, the torques of systems $\mathrm{X}$ and $\mathrm{Y}$ can be described by the following.

$$
\begin{aligned}
& \boldsymbol{T}_{1}: \boldsymbol{T}_{H_{1}}: \boldsymbol{T}_{4}=1:-i_{1 H_{1}}^{4}:\left(i_{1 H_{1}}^{4}-1\right) \\
& \boldsymbol{T}_{5}: \boldsymbol{T}_{\mathrm{H}_{2}}: \boldsymbol{T}_{8}=1:-i_{5 \mathrm{H}_{2}}^{8}:\left(i_{5 \mathrm{H}_{2}}^{8}-1\right)
\end{aligned}
$$

Where the $i_{1 H_{1}}^{4}$ is the speed ratio of $\mathrm{X}, i_{5 H_{2}}^{8}$ is the speed ratio of $\mathrm{Y}$, and $i_{18}^{4}$ is the total speed ratio.

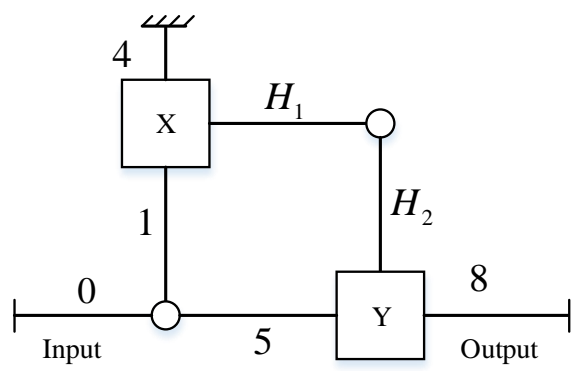

Fig. 4 The analysis diagram of power flow without power loss

Letting $i_{p}=i_{5 H_{2}}^{8}, i_{q}=1 / i_{1 H_{1}}^{4}$, then, the power of component 1 and 5 holds as Eq. (9):

$$
\frac{P_{1}}{P_{5}}=-i_{p} i_{q}
$$


Implied by Eq. (9), the power flow of traction drives can be divided into three cases shown in Table. 1 and Fig. 5.

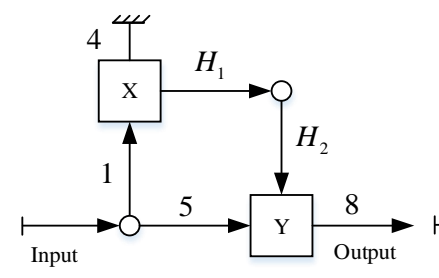

(a)

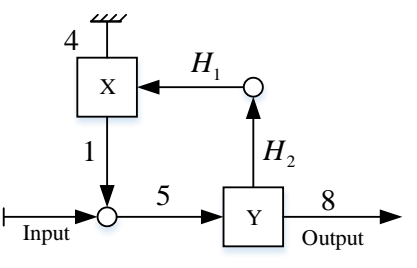

(b)

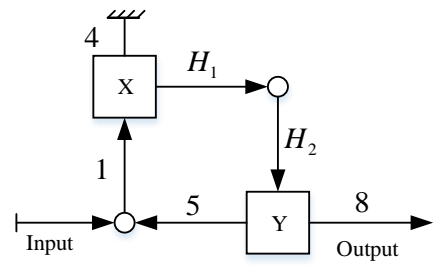

(c)

Fig. 5 Power flow diagrams: (a) Case 1 (b) Case 2 (c) Case 3

Table. 1 Power flow of traction drives

\begin{tabular}{c|c|c|c|c}
\hline \hline & $i_{p} i_{q}$ & $P_{1}$ & $P_{5}$ & $P_{8}$ \\
\hline Case 1 & $i_{p} i_{q}<0$ & $>0$ & $>0$ & $<0$ \\
\hline Case 2 & $0<i_{p} i_{q}<1$ & $<0$ & $>0$ & $<0$ \\
\hline Case 3 & $i_{p} i_{q}>1$ & $>0$ & $<0$ & $<0$ \\
\hline
\end{tabular}

Almost $i_{p}$ and $i_{q}$ complying with the guide that $0<i_{p}<1$ and $i_{p} i_{q}>1$ whether in researches and practices, the power flow, thus, coincides with case 3 in Fig. 5. For facilitating the process of research, a note is needed to make that the power flow direction diagram used in the follow-up research is case 3 if not explicitly explained.

II) Adjudging the driving-driven relationship of traction components without power loss in the conversion mechanism

Up to now, previous studies have indicated that the driving-driven relationship between transmission components may change because the angular velocity of the planetary carrier is varying before and after the conversion mechanism. So, it is wise to adjust the driving-driven relationship between traction components. As usual, the relation can be described by the power ratio that is defined as the ratio of the power in the original and conversion mechanism, just showing in Eq. (10). $\lambda_{i}$ is the power ratio of the traction parts, and $P_{i}$ or $P_{i}^{H_{1}}$ refers to the power of traction components in the original and conversion mechanism. Then, the results are displayed in Table. 2.

$$
\lambda_{1}=\frac{P_{1}^{H_{1}}}{P_{1}}>0 \quad \lambda_{2}=\frac{P_{5}^{H_{1}}}{P_{5}}>0 \quad \lambda_{3}=\frac{P_{8}^{H_{1}}}{P_{8}}<0
$$

Table. 2 Driving-driven relationships before and after conversion

\begin{tabular}{c|c|c|c}
\hline \hline & component 1 & component 5 & component 8 \\
\hline before conversion & driving & driven & driven \\
\hline after conversion & driving & driven & driving \\
\hline
\end{tabular}

III) Defining the creep at contact areas in the conversion mechanism

As mentioned above, the perfect rolling is not in existence because of some amount of slip that is regarded as the reason for the velocity difference on the contacting surfaces. Creep coefficient, however, is a normalized measure of the tangential slip velocity at the contact. Therefore, four creep coefficients $c_{r, i}(i=1,2,3,4)$ at different contact areas are defined as Eq. (11), and these four contact areas are separately specified as follows: the area of planetary rollers and the first stage inner raceway, the area of planetary rollers and the first stage outer raceway, the area of planetary rollers and the second stage inner raceway, the area of planetary rollers and the second stage outer raceway.

$$
\begin{array}{ll}
c_{r, 1}=\frac{\left|\omega_{1}^{\prime}\right| r_{1}-\left|\omega_{\text {orbit }}^{\prime}\right| r_{1}-\left|\omega_{\text {planet }, 1}^{\prime}\right| r_{2}}{\left|\omega_{1}^{\prime}\right| r_{1}-\left|\omega_{\text {orbit }}^{\prime}\right| r_{1}} \quad c_{r, 2}=\frac{\left|\omega_{\text {plane }, 1}^{\prime}\right| r_{3}-\left|\omega_{\text {orbit }}^{\prime}\right| r_{4}}{\left|\omega_{\text {planet }, 1}^{\prime}\right| r_{3}} \\
c_{r, 3}=\frac{\left|\omega_{1}^{\prime}\right| r_{1}-\left|\omega_{\text {orbit }}^{\prime}\right| r_{5}-\left|\omega_{\text {planet }, 2}^{\prime}\right| r_{6}}{\left|\omega_{1}^{\prime}\right| r_{5}-\left|\omega_{\text {orbit }}^{\prime}\right| r_{5}} \quad c_{r, 4}=\frac{\left|\omega_{\text {orbit }}^{\prime}\right| r_{8}-\left|\omega_{8}^{\prime}\right| r_{8}-\left|\omega_{\text {plane }, 2}^{\prime}\right| r_{7}}{\left|\omega_{\text {orbit }}^{\prime}\right| r_{8}-\left|\omega_{8}^{\prime}\right| r_{8}}
\end{array}
$$


IV) Modeling and estimating the actual speed ratio as well as global creep.

As shown in Fig. 6, the linear velocity of different traction components is described to explore the actual speed ratio, where the dotted and solid lines respectively represent the ideal and actual movement of each traction part. Moreover, the solid purple lines stand for the linear of the planetary carrier, and the black lines are the differential velocity at the contact areas. The creep coefficients, then, can also be defined as Eq. (12).

$$
c_{r, 1}=\frac{B_{1} B_{1}^{\prime}}{B_{1} G_{1}^{\prime}} \quad c_{r, 2}=\frac{E_{1} E_{1}^{\prime}}{E_{1}^{\prime} F_{1}^{\prime}} \quad c_{r, 3}=\frac{B_{2} B_{2}^{\prime}}{B_{2} G_{2}^{\prime}} \quad c_{r, 4}=\frac{H_{2}^{\prime} J_{2}^{\prime}}{H_{2}^{\prime} F_{2}^{\prime}}
$$

Similar to the process of the ideal speed ratio, the actual speed ratio can be written as Eq.(13) and Eq. (14)in the light of Eq. (12) and Fig. 6, where $i_{1 H_{1}}^{\prime}$ is the actual speed ratio of $\mathrm{X}$ and $i_{18}^{\prime}$ is the actual speed ratio.

$$
\begin{aligned}
& i_{1 H_{1}}^{\prime}=1+\frac{r_{2} r_{4}}{r_{1} r_{3}} \frac{1}{\left(1-c_{r, 1}\right)\left(1-c_{r, 2}\right)} \\
& i_{18}^{\prime}=\frac{1}{\frac{1}{i_{1 H_{1}}^{\prime}}-\frac{r_{5} r_{7}}{r_{6} r_{8}} \frac{1}{\left(1-c_{r, 3}\right)\left(1-c_{r, 4}\right)}\left(1-\frac{1}{i_{1 H_{1}}^{\prime}}\right)}
\end{aligned}
$$

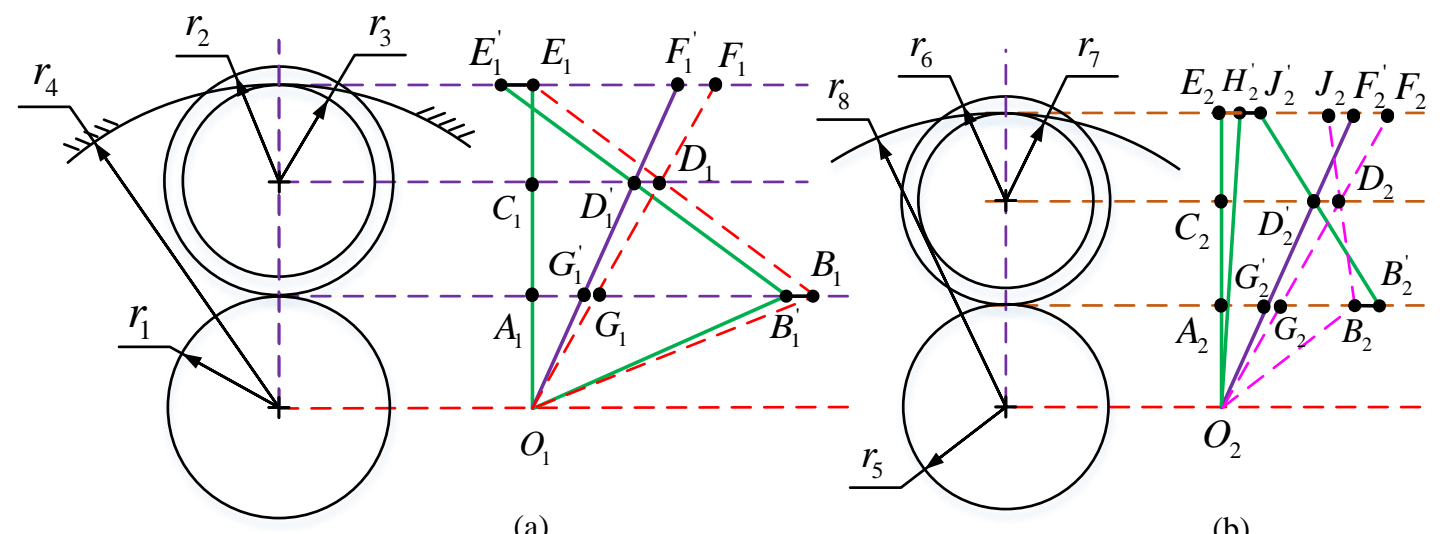

(a)

(b)

Fig. 6 (a) Actual angular velocity of the first stage (b) Actual angular velocity of the second stage

The global creep is adopted to illustrate the degree of difference between the ideal speed ratio and the actual speed ratio, which is defined as the differential speed ratio divided by the actual speed ratio.

$$
C_{r}=\frac{i_{18}^{\prime}-i_{18}}{i_{18}^{\prime}} \times 100 \%
$$

\subsubsection{Spin analysis}

Spin is widely found in traction drives and considered to be caused by different velocity distributions in the contact area, which is also the main factor for traction drive loss. It should be limited or eliminated in design due to its energy loss. Although the generatrix of the traction component has been redesigned to replace the circular generatrix and realizing zero-spinning theoretically ( $\mathrm{Li}$ et al., 2019; $\mathrm{Li}$ et al., 2015a, 2015b), it is also intractable for the new generatrix to be expressed by primary functions and manufactured. Therefore, the usual mean to limit the spin loss for improving efficiency is reducing the contact angle, which is also adapted in the proposed structure. According to the definition of spin velocity, the spin velocities generated at the contact areas are illustrated schematically in Fig. 7, where $\boldsymbol{s}_{i} \quad(i=1,2$, $3,4)$ is the unit vector of the normal of the contact point.

By Fig. 7, the relative angular velocity vectors of spin can be evaluated as follows: 


$$
\begin{aligned}
& \omega_{\text {spin }, 1}=0 \quad \omega_{\text {spin }, 3}=\left|\left(\omega_{4}^{\prime}-\omega_{\text {orbit }}^{\prime}-\omega_{\text {planet }, 1}^{\prime}\right) \sin \alpha\right| \boldsymbol{s}_{2} \\
& \omega_{\text {spin }, 3}=0 \quad \omega_{\text {spin }, 4}=\left|\left(\omega_{8}^{\prime}-\omega_{\text {orbit }}^{\prime}-\omega_{\text {plane }, 2}^{\prime}\right) \sin \beta\right| s_{4}
\end{aligned}
$$

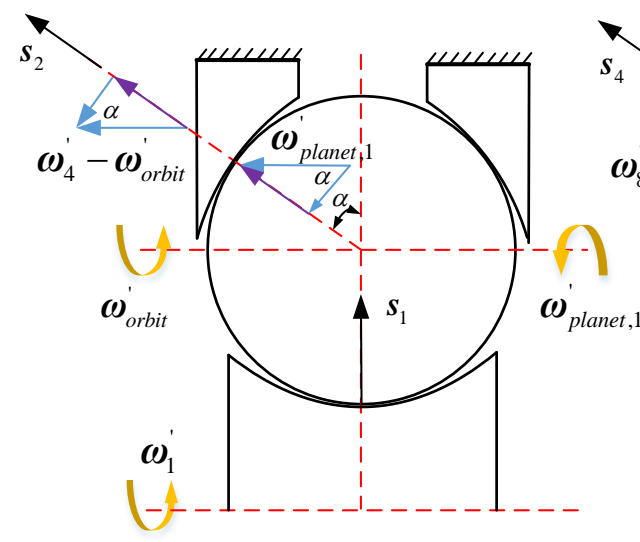

(a)

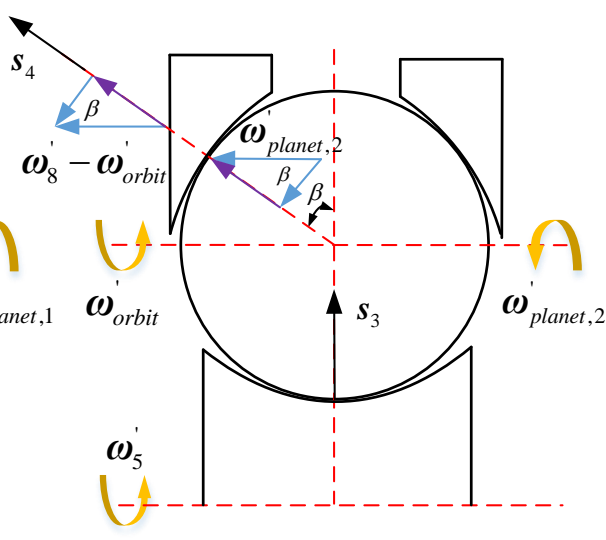

(b)

Fig. 7 (a) Spin velocity definitions of the first stage (b) Spin velocity definitions of the second stage

\subsection{Equilibrium equations description}

As can be seen from Fig. 8, the performances of forces and torques acted on each part are modeled when it is operating under steady-state conditions. An inertial coordinate system Oxyz is established according to the geometrical description of the structure, and ruling that the inertial coordinate system (a reference to other coordinate systems) is fixed in space. The $x$-axis coincides with the axis of input, the plane of yoz is perpendicular to the rotation axis, and the positive directions of the axis are determined by the right-hand rule.

In the free-body diagrams, $Q_{i}$ are the clamping force between the traction parts at contact area $i \quad(i=1,2,3,4)$, resulting from the contact pressure distribution. Similar but not identical to the friction drives, the traction drive forces $F_{t, i}$ come with the clamping force $Q_{i}$. Besides, the spin torques $T_{s, i}$ are the parasitical torque of spin, which work together with the angular velocity of spin to cause spin loss. $F_{b r \text {,fixed }}$ and $F_{b r \text {,out }}$ are the force of bearings and divided by the number of planetary rollers, considering the amount of them applied to each one.

For the planetary rollers of the first and second stage, forces and torques equilibrium equations can be derived from Fig. 8, and both of those are showing as Eq. (17) and Eq. (18).

$$
\begin{array}{r}
\left\{\begin{array}{c}
F_{t, 1}+2 F_{t, 2}-N_{\text {idler }, 1} \sin \xi_{1}=0 \\
F_{t, 1} r_{2}-2 F_{t, 2} r_{3}-2 T_{s, 2} \sin \alpha=0
\end{array}\right. \\
\left\{\begin{array}{c}
F_{t, 3}+2 F_{t, 4}-N_{\text {idler }, 2} \sin \xi_{2}=0 \\
F_{t, 3} r_{6}+2 T_{s, 4} \sin \beta-2 F_{t, 4} r_{7}=0
\end{array}\right.
\end{array}
$$

The equilibrium of the inner rings and idlers leads to the following equations:

$$
\begin{aligned}
& n_{p, 1} F_{t, 1} r_{1}-n_{p, 2} F_{t, 3} r_{5}=T_{i n} \\
& n_{p, 1} N_{i d l e r, 1} \sin \tau_{1}-n_{p, 2} N_{\text {idler }, 2} \sin \tau_{2}=0
\end{aligned}
$$

As illustrated in (Loewenthal, 1986), the normal force between traction components is provided by the loading cam, which is a mechanism that employs balls to create axial load. In Fig. 8(a), $N_{t}$ is the axial and tangential force of a single loading roller, which changes with external torque load $T_{l o a d}$, and $r_{l}$ is the contact radius of loading rollers from contact point to input axis. Moreover, $F_{a}$ and $T_{\text {shell }}$ are the axial force and torque of the shell. 

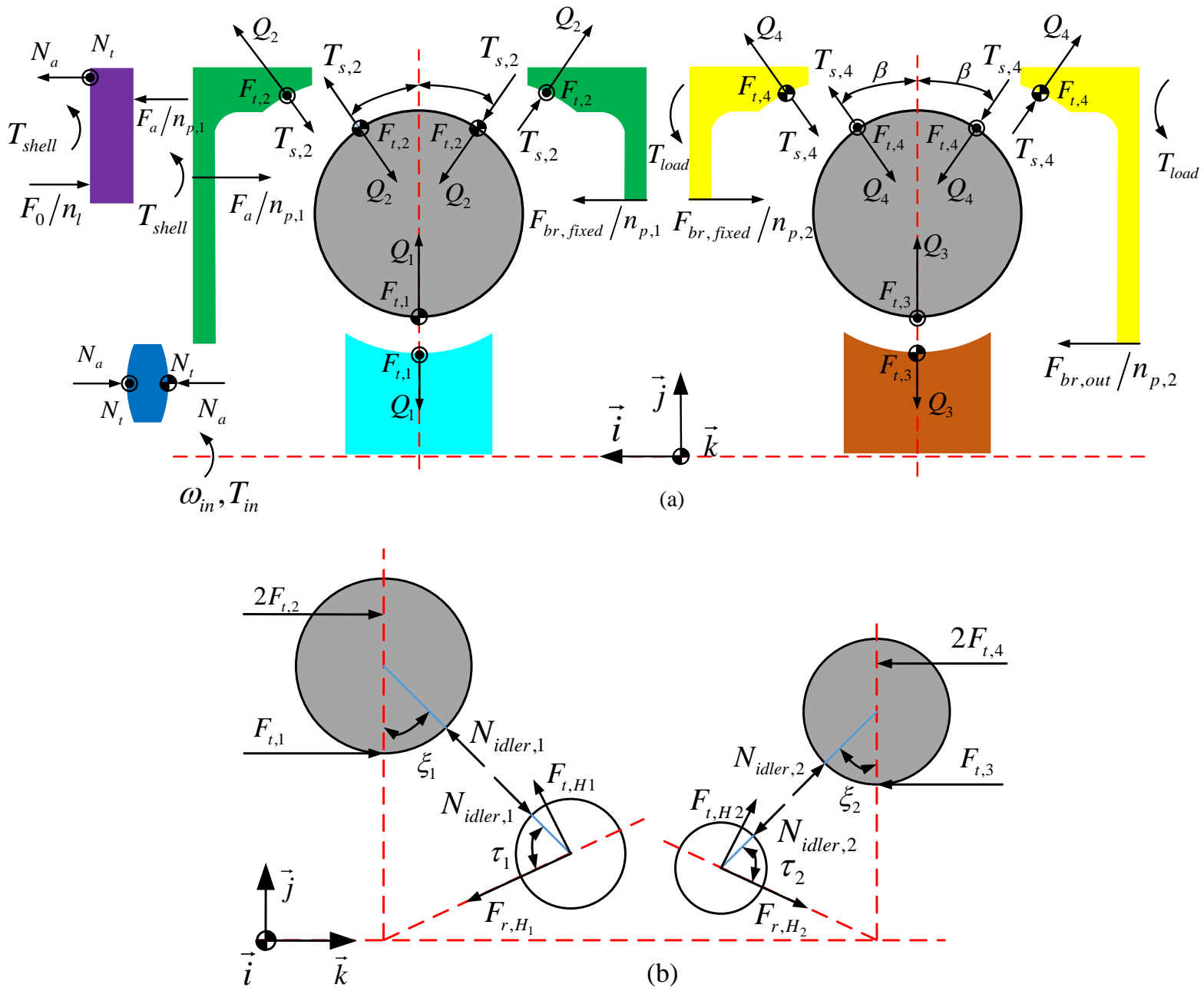

Fig. 8 (a) Free-body diagrams of main traction components and loading cam (b) Free-body diagrams of the planet rollers and idlers The symbol $\otimes$ is used for pointing away from the reader, and $\odot$ is used for pointing towards the reader.

By Fig. 8, clamping forces at contact areas can be expressed by Eq. (21), where $F_{0}$ is the preload.

$$
\left\{\begin{array}{l}
N_{t}=\frac{n_{p, 1} F_{t, 2} r_{4}}{n_{l} r_{l}} \\
F_{a}=\frac{N_{t}}{n_{l} \tan \delta}+F_{0} \\
Q_{1}+N_{\text {idler }, 1} \cos \xi_{1}=2 Q_{2} \cos \alpha \\
Q_{2}=\frac{F_{a}}{\sin \alpha} \\
Q_{3}+N_{\text {idler }, 2} \cos \xi_{2}=2 Q_{4} \cos \beta \\
Q_{4}=\frac{F_{a}}{\sin \beta}
\end{array}\right.
$$

\section{Efficiency}

\subsection{Efficiency of contact areas}

Usually, longitudinal slip, occurring at the ellipse contact areas, causes an offset of the spin pole from the center of the contact, and spin loss generates when power is transferred (Jeremy and Don, 2003; Loewenthal, 1986). At this moment, speed differences begin to appear between the ideal and actual, resulting from the change of the actual contacted point by the offset. Therefore, a function between the offset of the spin pole and creep is worth studying, which provides an important opportunity to advance the understanding of the contact acting.

Referring to the inertial coordinate system, the local coordinate systems $O x_{i}^{\prime} y_{i}^{\prime} z_{i}^{\prime}(i=1,2,3,4)$ are also built to study 
the function between the spin pole and creep, where the axis $x_{i}^{\prime}$ coincides with the $z$ axis and the planes of $y_{i}^{\prime} o z_{i}^{\prime}$ is formed by the normal and tangent lines of the contact point. The movements of contacted areas 2 and 4 in the conversion mechanism are shown in Fig. 9. The dashed and solid represent the ideal and actual respectively, and $m_{i}$ represents the offset of the spin pole at the ellipse contact area $i$.

Combining Eq. (11) and Fig. 9, the relationship between the spin pole offset and creep can be founded as Eq. (22).

$$
c_{r, 2}=1-\frac{r_{4}}{r_{3}} \cdot \frac{r_{3}-m_{2} \sin \alpha}{r_{4}-m_{2} \sin \alpha} \quad c_{r, 4}=1-\frac{r_{8}}{r_{7}} \cdot \frac{r_{7}-m_{4} \sin \beta}{r_{8}-m_{4} \sin \beta}
$$

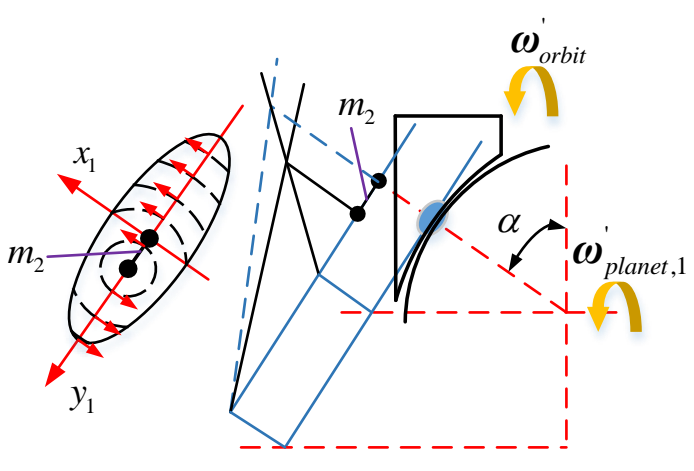

(a)

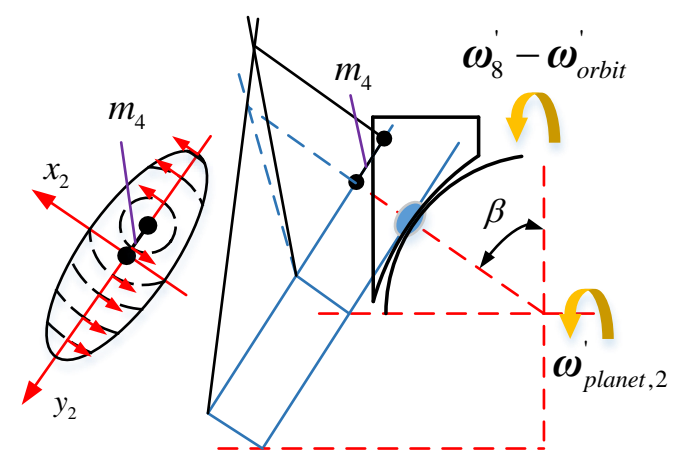

(b)

Fig. 9 (a) The spin pole offset at contact area 2 (b) The spin pole offset at contact area 4

The traction force and spin torque can be estimated by Eq. (23) and Eq. (24) based on the Hertz contact theory, where $a_{i}$ and $b_{i}$ are the semi-major axis and the semi-short axis of the contact ellipses $\Omega_{i} \quad(i=1,2,3,4)$.

$$
\begin{aligned}
& F_{t, i}=\frac{3 Q_{i}}{2 \pi a_{i} b_{i}} \oiint_{\Omega_{i}} \mu \sqrt{1-\left(\frac{y}{a_{i}}\right)^{2}-\left(\frac{x}{b_{i}}\right)^{2}} \frac{y-m_{i}}{\sqrt{x^{2}+\left(y-m_{i}\right)^{2}}} d x d y \\
& T_{s, i}=\frac{3 Q_{i}}{2 \pi a_{i} b_{i}} \oiint_{\Omega_{i}} \mu \sqrt{1-\left(\frac{y}{a_{i}}\right)^{2}-\left(\frac{x}{b_{i}}\right)^{2}} \sqrt{x^{2}+\left(y-m_{i}\right)^{2}} d x d y
\end{aligned}
$$

By Eq. (16) and Eq. (24), the power loss $P_{\text {loss }, i}$ and the efficiency of the contact area $\eta_{i}$ lead to the following, where $P_{i n, i}$ and $P_{o u t, i}$ are the input and output power at the contact area.

$$
\begin{aligned}
& P_{\text {loss }, i}=T_{s, i}\left|\omega_{\text {spin }}^{\prime}\right| \\
& \eta_{i}=\frac{P_{\text {in }, i}-P_{\text {loss }, i}}{P_{\text {in }, i}} \text { or } \eta_{i}=\frac{P_{\text {out }, i}}{P_{\text {out }, i}+P_{\text {loss }, i}}
\end{aligned}
$$

\subsection{Overall efficiency}

Understanding the link between efficiency at the contact areas and the efficiency of a single traction system will help us examine the overall efficiency. Hence, this section makes a major contribution to research on the relationship by demonstrating the structure of the $2 \mathrm{~K}-\mathrm{H}$ gear train indicated in Fig. 10, which is similar to the equivalent structure of the proposed one. In the procedure of analysis, the term $\eta_{c, i}$ ( $\left.i=\mathrm{I}, \mathrm{II}\right)$ will be used to refer to the efficiency of the contact points, and the symbols of $a$ to $d$ and $H$ are the transmission components in the $2 \mathrm{~K}-\mathrm{H}$ gear train.

From the graph above, we can see that the efficiency of a single traction system can be expressed as Eq. (27), where $\eta_{e f}^{g}$ and $i_{e f}^{g}$ are the efficiency and speed ratio from component $e$ to $f$ with component $g$ fixed (the symbols of $e, f, g$ stand for the transmission component in $2 \mathrm{~K}-\mathrm{H}$ gear train), and $\psi^{H}$ is the sum of the meshing loss coefficient. 


$$
\begin{aligned}
& \eta_{a H}^{b}=\eta_{H a}^{b}=1-\frac{\psi^{H}}{1+\left|i_{b a}^{H}\right|} \quad \eta_{b H}^{a}=\eta_{H b}^{a}=1-\frac{\psi^{H}}{1+\left|i_{a b}^{H}\right|} \quad \eta_{a b}^{H}=\eta_{b a}^{H}=1-\psi^{H} \\
& \psi^{H}=\sum\left(1-\eta_{c, i}\right) \quad(i=\mathrm{I}, \Pi)
\end{aligned}
$$

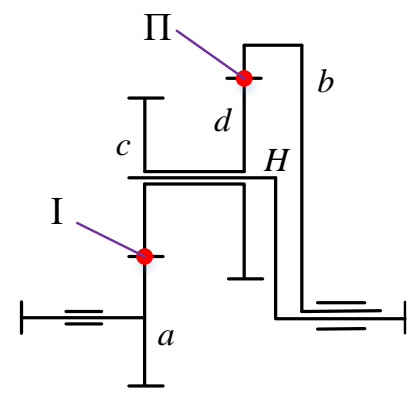

Fig. 10 Efficiency analysis diagram of $2 \mathrm{~K}-\mathrm{H}$ gear train

For the traction drive in Fig. 1, the efficiency expression of a single traction system is similar to Eq. (27) and can also be got by substituting the transmission components with traction components

$$
\begin{cases}\eta_{1 H_{1}}^{4}=\eta_{H_{1} 1}^{4}=1-\frac{\sum\left(1-\eta_{i}\right)}{1+\left|i_{41}^{H_{1}}\right|} \quad(i=1,2) \\ \eta_{58}^{H_{2}}=\eta_{85}^{H_{2}}=1-\sum\left(1-\eta_{i}\right) \quad(i=3,4) \\ \eta_{H_{2} 8}^{5}=\eta_{8 H_{2}}^{5}=1-\frac{\sum\left(1-\eta_{i}\right)}{1+\left|i_{58}^{H_{2}}\right|} \quad(i=3,4)\end{cases}
$$

As the existing traction drives rotating around a fixed axis, taking half or full toroidal CVT (Verbelen et al., 2018) and NuVinci (Tomaselli et al., 2020) for example, the overall efficiency can be expressed as the product of speed efficiency and torque efficiency easily. However, contrasted to the fixed-axis traction drive systems, there is much less research about the planetary traction drives. A new approach, thus, is introduced to establish the efficiency model, which depends on the power flow to provide a novel idea to establish the efficiency model. The analysis diagrams of power flow are shown in Fig. 11, where $P_{i n, i}$ and $P_{\text {out }, i}$ ( $i$ represents the traction component) are the input and output power relative to the system of $\mathrm{X}$ and $\mathrm{Y}$ respectively.

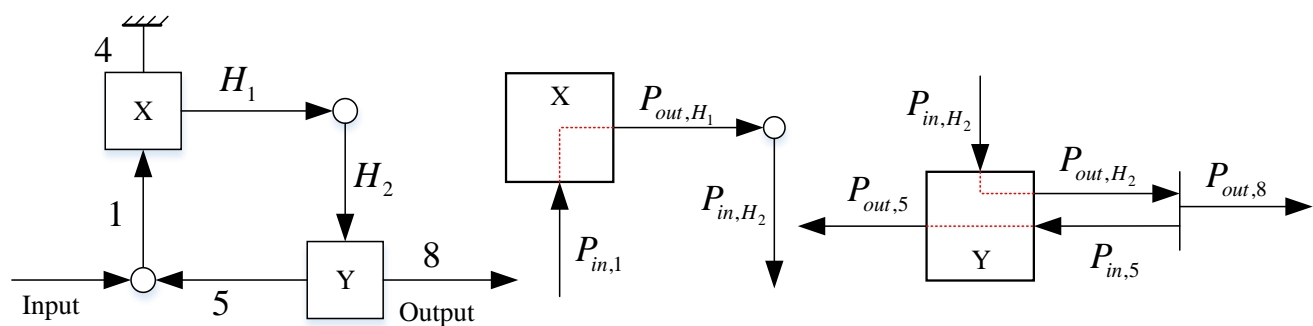

(a)

(b)

(c)

Fig. 11 (a) Power flow of AL-BTDR $\quad$ (b) Power flow of system X (c) Power flow of system Y

By Fig. 11, the power through the systems X and Y follow:

$$
\left\{\begin{array}{l}
P_{\text {out }, H_{1}}=-P_{i n, 1} \eta_{1 H_{1}}^{4} \\
P_{i n, H_{2}}=-P_{\text {out }, H_{1}} \eta_{H_{1} H_{2}} \\
P_{i n, H_{2}}=T_{H_{2}} \omega_{H_{2}} \\
T_{H_{1}}=-T_{H_{2}}
\end{array}\right.
$$




$$
\left\{\begin{array}{l}
P_{\text {out }, 8}=P_{\text {out }, H_{2}}+P_{i n, 5} \\
P_{\text {out }, H_{2}}=-P_{\text {in }, H_{2}} \eta_{H_{2} 8}^{5} \\
P_{i n, 5}=-P_{\text {out }, 5} / \eta_{85}^{H_{2}} \\
P_{\text {in }}=P_{\text {out }, 5}+P_{\text {in }, 1} / \eta_{1}
\end{array}\right.
$$

Combining Eq. (29) and Eq. (30), the overall efficiency can be written as Eq. (31).

$$
\eta=\frac{\eta_{1 H_{1}}^{4} \eta_{H_{1} H_{2}} \eta_{1}\left(C i_{q}^{\prime} \eta_{H_{2} 8}^{5} \eta_{85}^{H_{2}}+1\right)}{\eta_{85}^{H_{2}}\left(\eta_{1 H_{1}}^{4} \eta_{H_{1} H_{2}} \eta_{1}+C i_{q}^{\prime}\right)}
$$

Where $C=\frac{\eta_{85}^{H_{2}}-i_{18}^{\prime}}{i_{18}^{\prime} i_{q}^{\prime} \eta_{H_{2} 8}^{5} \eta_{85}^{H_{2}}-\eta_{85}^{H_{2}}}, \quad i_{q}^{\prime}=\frac{1}{i_{1 H 1}^{\prime}}$.

Similar to the analysis process above, the overall efficiency can also be expressed by Eq. (32) and Eq. (33) if the power flow shows as Fig. 5(b) and Fig. 5(c).

$$
\begin{aligned}
& \eta=\frac{\eta_{1 H_{1}}^{4} \eta_{H_{1} H_{2}} \eta_{5}\left(A i_{q}^{\prime} \eta_{H_{2} 8}^{5}+\eta_{58}^{H_{2}}\right)}{\eta_{1 H_{1}}^{4} \eta_{H_{1} H_{2}}+A i_{q}^{\prime} \eta_{5}} \\
& \eta=\frac{\eta_{58}^{H_{2}} \eta_{8 H_{2}}^{5} \eta_{5}+B i_{q}^{\prime} \eta_{5}}{B i_{q}^{\prime} \eta_{H_{1} 1}^{4} \eta_{H_{2} H_{1}} \eta_{8 H_{2}}^{5} \eta_{5}+\eta_{8 H_{2}}^{5}}
\end{aligned}
$$

Where $A=\frac{1-i_{18}^{\prime} \eta_{58}^{H_{2}}}{i_{18}^{\prime} i_{q}^{\prime} \eta_{H_{2} 8}^{5}-1}, \quad B=\frac{\eta_{8 H_{2}}^{5}-i_{18}^{\prime} \eta_{58}^{H_{2}}}{i_{18}^{\prime} i_{q}^{\prime}-\eta_{8 H_{2}}^{5}}$.

\section{An illustrative example and discussion}

There is an important guiding significance for mathematical models in terms of performance analysis, design, and optimization. The efficiency performance has long been a great interest in a wide range of traction drive fields, which can be adopted to evaluate the quality of the traction drive system. Then, the work of this part mainly investigates the performance of overall efficiency and its influencing factors based on the efficiency model with the parameters reported in Table. 3. At the same time, the overall calculation process for estimating the performance of traction drive reducer can be shown in Fig. 12.

Table. 3 Geometric parameters

\begin{tabular}{l|l}
\hline \hline Geometric properties & value \\
\hline roll radius $(\mathrm{mm})$ & $R_{\text {plant }, 1}=19.844, R_{\text {plant }, 2}=19.844$ \\
\hline inner raceway $(\mathrm{mm})$ & $R_{\text {in }, 1}=12.019, R_{\text {in }, 2}=12.019$ \\
\hline outer raceway $(\mathrm{mm})$ & $R_{\text {out }, l}=25.797, R_{\text {out }, 2}=25.797$ \\
\hline distance between inner center and datum $(\mathrm{mm})$ & $R_{\text {ind, },}=33, R_{\text {ind }, 2}=31$ \\
\hline idler radius $(\mathrm{mm})$ & $r_{\text {idler }, 1}=11, r_{\text {idler }, 2}=10.141$ \\
\hline contact angular between roll and outer $\operatorname{ring}\left({ }^{\circ}\right)$ & $\alpha=10, \beta=13$ \\
\hline number of first stage roller & $n_{p, l}=3$ \\
\hline number of second stage roller & $n_{p, 2}=3$ \\
\hline number of loading roller & $n_{l}=8$ \\
\hline angular of loading slope $\left({ }^{\circ}\right)$ & $\delta=28$ \\
\hline ideal speed ratio & $i_{18}=35.05$ \\
\hline
\end{tabular}




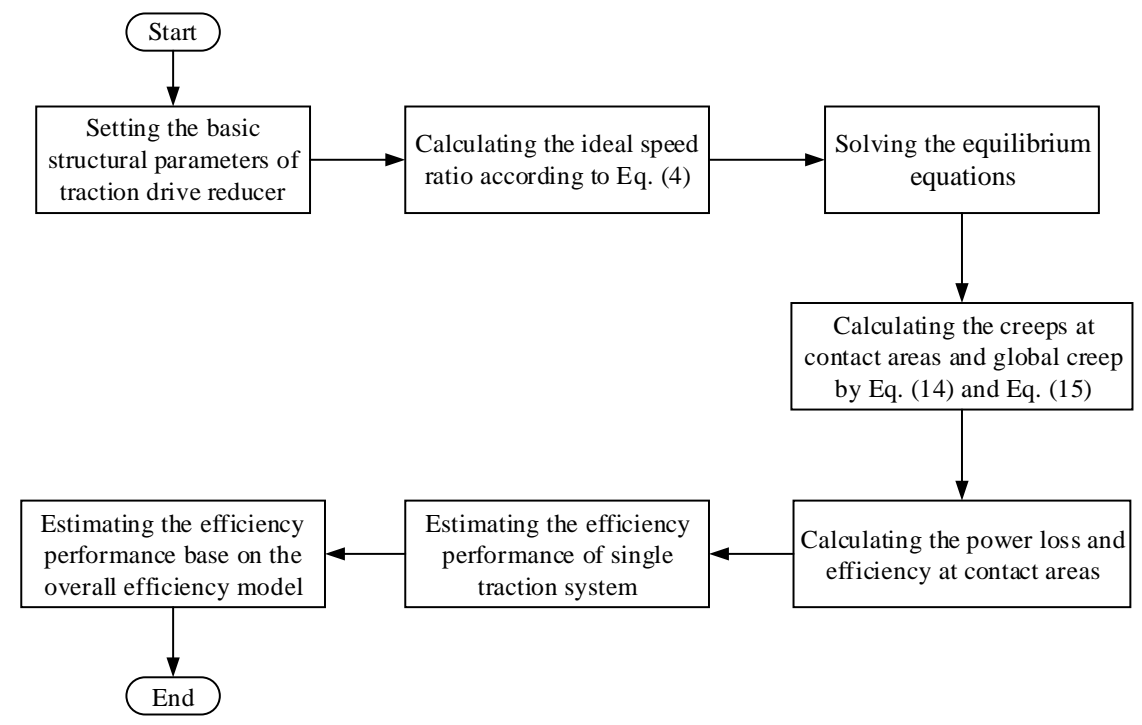

Fig. 12 The flowchart of calculating process

Studies over the past have provided important information on the investigation of efficiency. Since the contact areas at contact points 1 and 3 are parallel to the axes of traction parts, it is potential for them to realize zero-spin loss in theory, thus, the power loss and efficiency at contact points 2 and 4 are mainly studied in this paper. It is convenient to redefine some dimensionless terms that enable us to better understand the trend of change before the formal analysis.

The first term in this section sought to determine is spin coefficients that are a function of spin velocities and the relative input speed.

$$
\sigma_{s, 2}=\frac{\boldsymbol{\omega}_{s p i n, 2} \cdot \boldsymbol{s}_{2}}{\left|\boldsymbol{\omega}_{3}^{\prime}\right|} \quad \sigma_{s, 4}=\frac{\boldsymbol{\omega}_{s p i n, 4} \cdot \boldsymbol{s}_{4}}{\left|\boldsymbol{\omega}_{7}^{\prime}\right|}
$$

Similarly, the equivalent traction coefficients can be also defined as the tangential contact forces responsible for the torque transmissions dividing by the related normal contact forces:

$$
\mu_{2}=F_{t, 2} / Q_{2} \quad \mu_{4}=F_{t, 4} / Q_{4}
$$

Besides, the relative spin momentum coefficients are defined as Eq. (36), which can be utilized to describe the proportion between the effective torque and the spin torque.

$$
\kappa_{2}=\frac{T_{s, 2}}{F_{t, 2} r_{4}} \quad \kappa_{4}=\frac{T_{s, 4}}{F_{t, 4} r_{8}}
$$

Where $\sigma_{s, 2}, \mu_{2}$, and $\kappa_{2}$ are the spin coefficient, equivalent traction coefficient, and the relative spin momentum coefficient at contact area 2 respectively; as the same way, $\sigma_{s, 4}, \mu_{4}$, and $\kappa_{4}$ are the spin coefficient, traction coefficient, and the relative spin momentum coefficient at contact area 4 .

Figure 12 provides the intercorrelations between output torque and the spin coefficients, creep of contact areas, global creep, and overall efficiency, which are obtained by the efficiency model (Eq. (32)) and operated under the situation that input speed is constant and equal to $3000 \mathrm{rpm}$. According to (Jeremy and Don, 2003), spin velocity, in theory, is determined purely by the geometry of the system and the speed of the rollers. As shown in Fig. 13 (a), yet, it can be seen that the spin coefficient of the contact area 2 increases with the development of output torque, while the contact area 4 is the opposite. A possible explanation for these results may be the offsets of the spin pole that result in an increment on the actual contact angles of the first stage and a decrease on the second stage, which can be seen from Fig. 9, thus leading to 
the variation of geometric parameters during the actual operating. From Fig. 13 (b), it is apparent that the creeps gradually increase from zero within contact areas 2 and 4 to meet the traction with an escalation in the output torque, which also causes an increment to the global creep. However, the global creep not start from zero for the reason that the creeps within contact areas 1 and 3 are assumed to be $0.2 \%$ (not shown in Fig. 13 (b)) by referring (Ai, 2001a, 2010b)), which is utilized to characterize the speed difference of traction parts in contact areas 1 and 3. Moreover, another finding also can get that the creeps of contact areas 2 and 4 differ from the global creep by almost an order of magnitude.

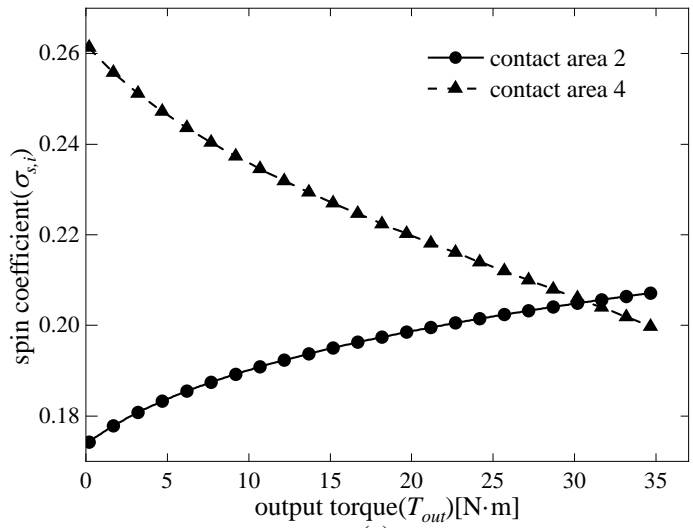

(a)

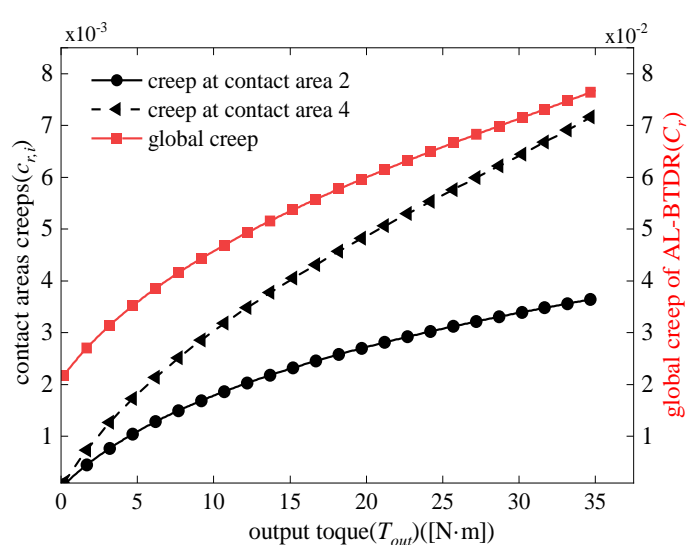

(b)

Fig. 13 (a) Spin coefficients as a function of output torque (b) Contact areas creeps and global creep as a function of output torque
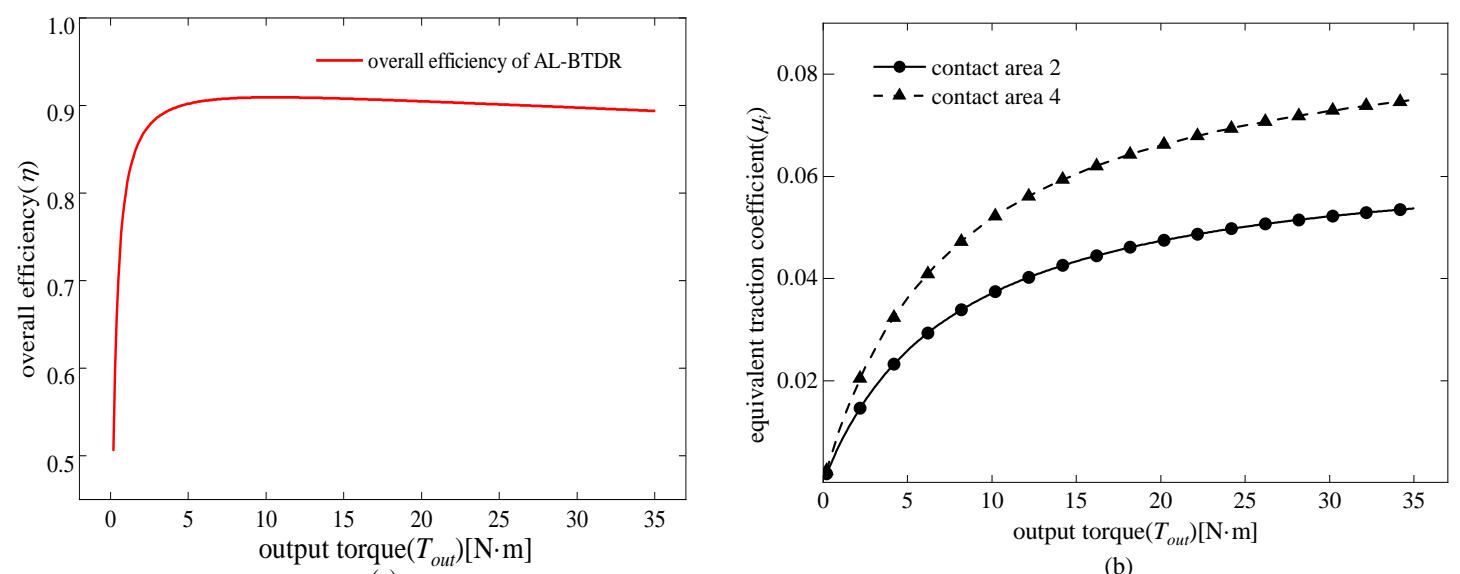

(a)

(b)

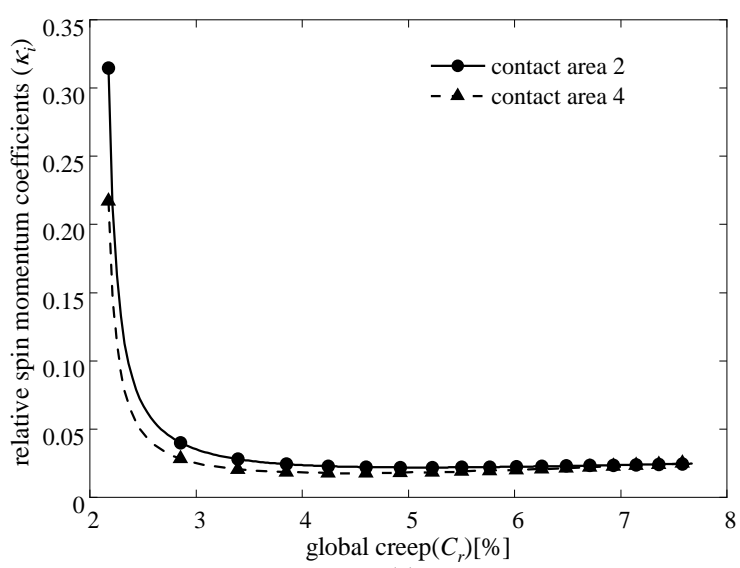

(c)

Fig. 14 (a) Overall efficiency as a function of output torque (b) Traction coefficients as a function of output torque (c)

Relative spin momentum coefficients as a function of global creep 
As can be seen from Fig. 14(a), the overall efficiency is described as a function of output torque. What is interesting is that the overall efficiency is lower at the original output torque and increases with output torque increasing, then decline slowly. During the operation, the clamping force is provided by the preload and the loading cam, and the proportions of those will vary with output torque. At low output torque, the preload accounts for a higher proportion, and the clamping forces exceed the required, thus, the overload occurs at contact areas. Fig. 14(b) also illustrates the phenomenon by the traction coefficients that are small during lower output torque. At the same time, the trend of relative spin momentum coefficients is opposite with overall efficiency, which means that the spin torque has a heavy impact on efficiency when the power of output is lower. It is obvious that the clamping force will increase as the output torque increases, which reduces the phenomenon of overload to some extent and lead to the rise of overall efficiency. For the further output torque, the larger clamping forces can be got by the loading cam, which can avoid the excessive growth of global creep and slipping failure. It also can be seen from Fig. 14(c) that the relative spin momentum coefficients increase slowly as the global increases. Therefore the overall efficiency can slowly decline and maintain a high level with a peak efficiency of $91 \%$.

In order to study the influence of loading mode on efficiency, this paper investigates the efficiency performance under constant loading (Novellis et al., 2012; Tomaselli et al., 2020) and adaptive loading. For better illustrating the distinctions between the two loading methods, the spin offset coefficient is defined as the ratio of the spin pole offset to the major semi-axis of the elliptical contact area.

$$
s r_{i}=\frac{\left|m_{i}\right|}{a_{i}} i=(2,4)
$$

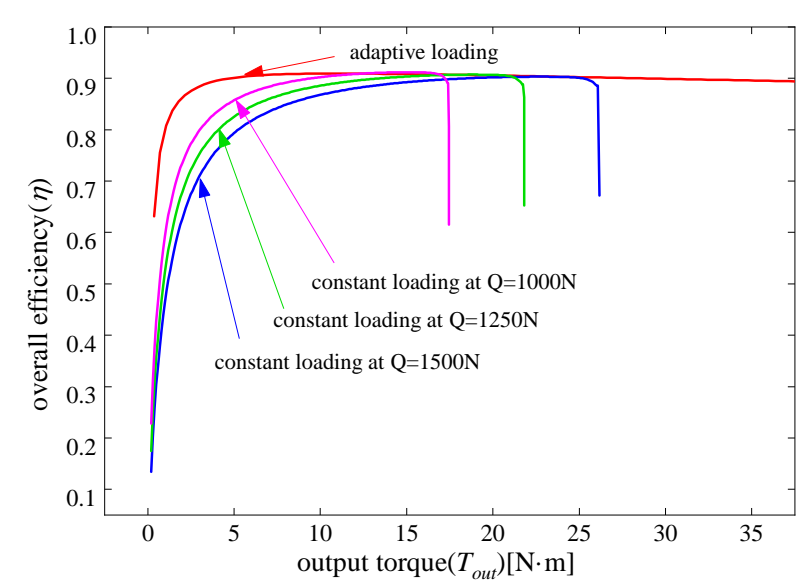

(a)

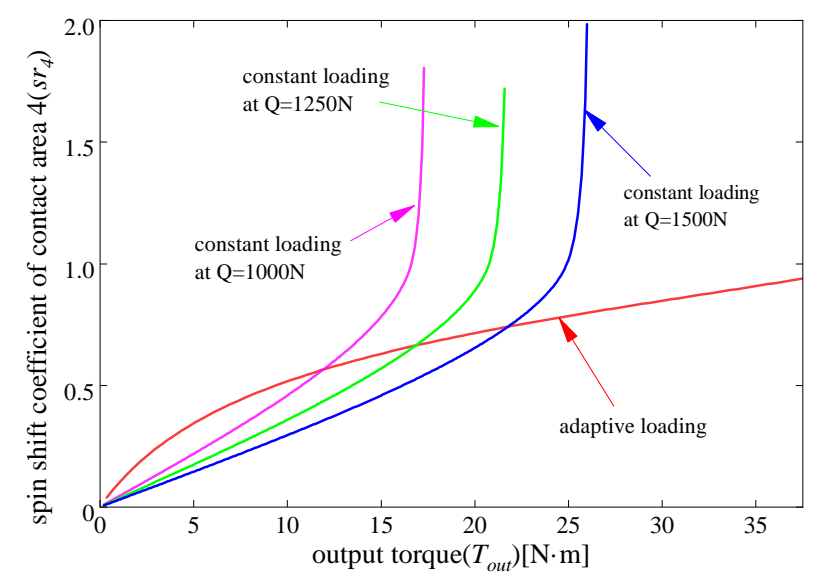

(c)

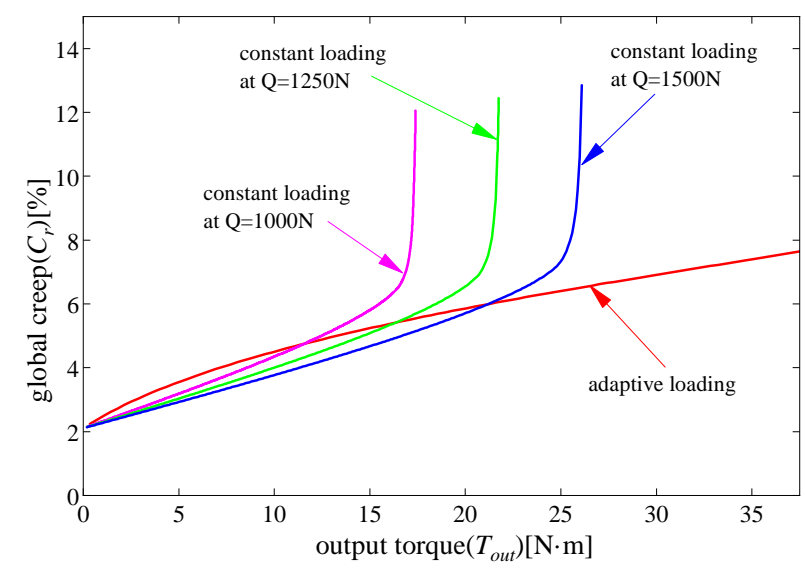

(b)

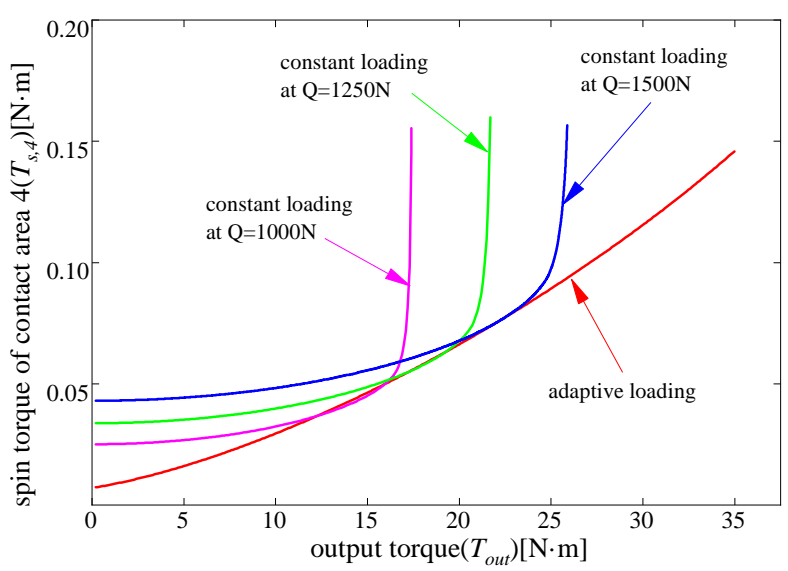

(d)

Fig. 15 Performances under adaptive loading and constant loading: (a) Overall efficiency (b) Global creep (c) Spin shift coefficient at contact area 4 (d) Spin torque at contact area 4 
The results of the constant loading shown in Fig. 15 are obtained under the following hypotheses: input speed is constant and equal to $3000 \mathrm{rpm}$, and the clamping force $Q_{2}$ is fixed at $1000 \mathrm{~N}, 1250 \mathrm{~N}$, and $1500 \mathrm{~N}$. Moreover, the calculating is performed in this way: the output torque is set as a torque vector with a certain step length, and simulations start with a minimal amount of torque. Then, all models of the kinematics, equilibrium equations, and overall efficiency are calculated at different output torque. What's more, a smaller step than the former is adopted to evaluate the efficiency performance when the global creep is about to increase sharply.

Figure 14 (a-d) show the overall efficiency, global creep, spin offset coefficient, and spin torque as a function of output under the two loading modes. To make the image more concise, this paper only studies the spin offset coefficient and spin torque at contact area 4, which are more evident than contact area 2. By Fig. 15 (a), the trend of efficiency under constant loading is similar to the one under adaptive loading during the initial output torque. However, the efficiency is lower under constant loading for the reason that the constant clamping force is more extravagant than the adaptive loading, thus, resulting in a severe overload at the contact areas than the acting under adaptive loading. According to Eq. (23), a higher clamping force is beneficial to reduce the spin pole offset and the creeps at contact areas. Therefore, the global creep in Fig. 15 (b) and spin shift coefficient at the contact area 4 in Fig. 15 (c) are lower than the one under adaptive loading during the stage of lower output torque. Yet, the spin torque will increase by referring Eq. (24), which can be seen from Fig. 15 (d). As expected, the larger the constant loading is, the lower the overall efficiency will be.

Besides, there is a significant difference between the two loading methods. A sharp drop occurs in the overall efficiency during the constant loading that also appears in (Verbelen et al., 2017). Moreover, the smaller the loading force is, the earlier the efficiency will drop. This discrepancy could be attributed to the global creep and spin torque that is described in Fig. 15 (b-d). As the figures showed, global creep, spin shift coefficient, and spin torque at contact area 4 will increase sharply with a small increase in torque under constant loading. Then, the spin loss within the contact area 4 will increase dramatically caused by the sharp increase of global creep (affected by the spin shift coefficient at contact area 4) and spin torque, which leads to the so-called sliding failure phenomenon in the macro view and the decline of overall efficiency. Contrasting to the mode of constant loading, global creep, and spin torque increases smoothly under adaptive loading, and the overall efficiency will not drop dramatically. Thus, efficiency performs better under adaptive loading than constant loading during the whole traction process.

\section{Conclusions}

In this paper, a new type of adaptive loading ball traction drive reducer is presented and studied, and the following conclusions can be drawn:

- A method founded on power flow and equal linear velocity is developed to establish the mathematical models of the ideal and actual speed ratio (Eq. (4) and Eq. (14)) of planetary traction drive for the first time.

- Depending on the power flow direction of transmission components, a new approach is introduced to establish the overall efficiency models (Eq. (31)-Eq. (33)) of different power flow situations.

- Based on the overall efficiency models, the performances of global creep and overall efficiency are studied (shown in Fig. 14). The research shows that the creeps at the contact areas increase as the output torque. Furthermore, overall efficiency is similar to previous and can maintain a high level with a maximum efficiency of $91 \%$ because the clamping force can be satisfied through the adaptive loading mechanism.

- The overall efficiency performances under adaptive loading and constant loading are evaluated and discussed. A gigantic difference between the two loading modes is that the overall efficiency will drop sharply under constant loading caused by excessive global creep and spin torque, which can lead to the so-called sliding failure phenomenon in the macro view. Furthermore, the smaller the loading force is, the earlier the efficiency will drop. By contrast, the overall efficiency performs better under adaptive loading than constant loading during the whole traction process.

What needed to be emphasized is that all results in this manuscript are obtained from theoretical analysis. During the process of analysis, some simplifications that are also adopted by major research are carried out here: (a) ignoring the influence of temperature, (b) the power loss is substituted by spin loss (primary element of power loss). Although these can simplify our work, there will be some distinction between the calculated results and the real value. In the future, we 
will re-establish the overall efficiency models with temperature and other forms of power loss considered, which is more helpful for us to analyze the efficiency of traction drive.

\section{Acknowledgments}

This research is supported by the National Natural Science Foundation of China (Grant No. 92048201). The authors thank the reviewer for his/her valuable comments on the manuscript.

\section{References}

Afrabandpey, A. and Ghariblu, H., Performance Evaluation of Ball CVT and Comparison with Half Toroidal CVT, International Journal of Automotive Technology, Vol. 19, No. 3 (2018), pp. 547-557.

Ai, X., Development of Zero-Spin Planetary Traction Drive Transmission: Part 1 -Design and Principles of Performance Calculation, Journal of Tribology, Vol. 124, No. 2 (2001a), pp. 386-391.

Ai, X., Development of Zero-Spin Planetary Traction Drive Transmission: Part 2 - Performance Testing and Evaluation, Journal of Tribology, Vol. 124, No. 2 (2001b), pp. 392-397.

Ai, X., Matthew, W. and David, L., Development of Friction Drive Transmission, Journal of Tribology, Vol. 127, No. 4 (2005), pp. 857-864.

Akehurst, S., Parker, D. A. and Schaaf, S., CVT Rolling Traction Drives-A Review of Research Into Their Design, Functionality, and Modeling, Journal of Mechanical Design, Vol. 128, No. 5 (2005), pp. 1165-1176.

Akehurst, S., Parker, D. A. and Schaaf, S., Dynamic Modeling of the Milner Continuously Variable Transmission—The Basic Kinematics, Journal of Mechanical Design, Vol. 129, No. 11 (2006), pp. 1170-1178.

Baejin, K., Sehoon, O. and Seongyoung, P., Manufacture of elastic composite ring for planetary traction drive with silicon rubber and carbon fiber, Composite Structures, Vol. 66, No. 1 (2004), pp. 543-546.

Bertini, L., Carmignani, L. and Frendo, F., Analytical model for the power losses in rubber V-belt continuously variable transmission (CVT) (2014), pp. 289-306, Mechanism and Machine Theory.

Brassitos, E. and Jalili, N., Design and Development of a Compact High-Torque Robotic Actuator for Space Mechanisms, Journal of Mechanisms and Robotics 061002 (2017).

Chen, C., Power flow and efficiency analysis of epicyclic gear transmission with split power (2013), pp. 96-106, Mechanism and Machine Theory.

Chen, C. and Jorge, A., Virtual-Power Flow and Mechanical Gear-Mesh Power Losses of Epicyclic Gear Trains, Journal of Mechanical Design, Vol. 129, No. 1 (2006), pp. 107-113.

Chen, X., Wei, H., Deng, T., He, Z. and Zhao, S., Investigation of electromechanical coupling torsional vibration and stability in a high-speed permanent magnet synchronous motor driven system (2018), pp. 235-248, Applied Mathematical Modelling.

Cretu, O. S. and Glovnea, R. P., Traction Drive With Reduced Spin Losses, Journal of Tribology-transactions of The ASME, Vol. 125, No. 3 (2003), pp. 507-512.

Flugrad, D. R. and Qamhiyah, A. Z., A Self-Actuating Traction-Drive Speed Reducer, Journal of Mechanical Design, Vol. 127, No. 4 (2004), pp. 631-636.

Georgiev, N. and Burdick, J., Design and analysis of the bearingless planetary gearbox, Proceedings of the 2017 IEEE/RSJ International Conference on Intelligent Robots and Systems (IROS) (2017), pp.1987-1994.

Ghariblu, H., Behroozirad, A. and Madandar, A., Traction and Efficiency Performance of B all Type CVTs, International Journal of Automotive Engineering, Vol. 4, No. 2 (2014), pp. 738-748.

Itagaki, H., Ohama, K. and Raja Rajan, A. N., Method for estimating traction curves under practical operating conditions, Tribology International 105639 (2020).

Jeremy, C. and Don, M., The Design and Analysis of an Alternative Traction Drive CVT, JSAE ReviewSAE transactions, Vol. 112, No. 6 (2003), pp. 1051-1061.

Jean-Philippe, G. and Philippe, M., A model based on experimental data for high speed steel belt CVT, Mechanism and Machine Theory, Vol. 45, No. 11 (2010), pp. 1733-1744.

Jiang, P., Li, X. M., Guo, F. and Chen, J., Interferometry Measurement of Spin Effect on Sliding EHL, Tribology Letters, Vol. 33, No. 3 (2008), pp. 161-168. 
Kong, J., Kim, J., Kang, H.-Y. and Kim, C.-J., Slip Analysis on a Non-holonomic Continuously Variable Transmission Using Magic Formula, Proceedings of the AETA 2017-Recent Advances in Electrical Engineering and Related Sciences: Theory and Application (2018), pp.1003-1013.

Li, C., Li, H., Li, Q., Zhang, S. and Yao, J., Modeling, kinematics and traction performance of no-spin mechanism based on roller-disk type of traction drive continuously variable transmission (2019), pp. 278-294, Mechanism and Machine Theory.

Li, Q., Li , H., Yu, D. and Yao, J., A novel continuously variable transmission with logarithmic disc generatrix (2015a), pp. 147-162, Mechanism and Machine Theory.

Li, Q., Wu, J., Li, H. and Yao, J., A Mathematical Method for Eliminating Spin Losses in Toroidal Traction Drives (2015b), pp. 1-10, Mathematical Problems in Engineering.

Li, Q., Liao, M. and Wang, S., A Zero-Spin Design Methodology for Transmission Components Generatrix in traction drive Continuously Variable Transmissions, Journal of Mechanical Design 033301 (2017).

Li, X. M., Guo, F., Fan, B. and Yang, P., Influence of spinning on the rolling EHL films, Tribology International, Vol. 43, No. 11 (2010), pp. 2020-2028.

Loewenthal, S. H., Spin Analysis of Concentrated Traction Contacts, Journal of Mechanisms, Transmissions, and Automation in Design, Vol. 108, No. 1 (1986), pp. 77-84.

Loewenthal, S. H., Anderson, N. E. and Rohn, D. A., Evaluation of a High Performance Fixed-Ratio Traction Drive, Journal of Mechanical Design, Vol. 103, No. 2 (1981), pp. 420-420.

Mahanto, B. S., Sahoo, V. and Maiti, R., Effect of Cam Insertion on Stresses in Harmonic Drive in Industrial Robotic Joints (2018), pp. 432-439, Procedia Computer Science.

Matsuda, K., Goi, T., Tanaka, K., Imai, H., Tanaka, H. and Sato, Y., Study on high-speed traction drive CVT for aircraft power generation - Gyroscopic effect of the thrust ball bearing on the CVT, Journal of Advanced Mechanical Design, Systems, and Manufacturing, Vol. 11, No. 6 (2017), DOI: 10.1299/jamdsm.2017jamdsm0087.

Miyata, S. and Liu, D., Study of the Control Mechanism of a Half-Toroidal CVT during Load Transmission, Journal of Advanced Mechanical Design, Systems, and Manufacturing, Vol. 1, No. 3 (2007), pp. 346-357.

Novellis, L. D., Carbone, G. and Mangialardi, L., Traction and Efficiency Performance of the Double Roller Full-Toroidal Variator: A Comparison With Half- and Full-Toroidal Drives, Journal of Mechanical Design 071005 (2012).

Okuda, R., Okubo, K., Fujii, T., Sakagami, K. and Yagasaki, T., Slip behavior in pulley groove up to sliding slip at steady state and power transmitting efficiency on metal V-belt type cvt, Journal of Advanced Mechanical Design, Systems, and Manufacturing, Vol. 11, No. 6 (2017), DOI: 10.1299/jamdsm.2017jamdsm0086.

Qian, H.-M., Li, Y.-F. and Huang, H.-Z., Time-variant reliability analysis for industrial robot RV reducer under multiple failure modes using Kriging model, Reliability Engineering \& System Safety 106936 (2020).

Sharif, K. J., Evans, H. P., Snidle, R. W. and Newall, J. P., Modeling of Film Thickness and Traction in a Variable Ratio Traction Drive Rig, Journal of Tribology, Vol. 126, No. 1 (2004), pp. 92-104.

Tomaselli, M., Lino, P. and Carbone, G., Modelling and efficiency formulation of a planetary traction drive CVT, IFACPapersOnLine, Vol. 52, No. 5 (2019), pp. 411-416.

Tomaselli, M., Bottiglione, F., Lino, P. and Carbone, G., NuVinci drive: Modeling and performance analysis, Mechanism and Machine Theory 103877 (2020).

Tyreas, G. C. and Nikolakopoulos, P. G., Development and friction estimation of the Half-Toroidal Continuously Variable Transmission: A wind generator application (2016), pp. 63-80, Simulation Modelling Practice and Theory.

Verbelen, F., Derammelaere, S., Sergeant, P. and Stockman, K., A comparison of the full and half toroidal continuously variable transmissions in terms of dynamics of ratio variation and efficiency (2018), pp. 299-316, Mechanism and Machine Theory.

Verbelen, F., Derammelaere, S., Sergeant, P. and Stockman, K., Half toroidal continuously variable transmission: Tradeoff between dynamics of ratio variation and efficiency (2017), pp. 183-196, Mechanism and Machine Theory.

Yamamoto, T., Matsuda, K. and Hibi, T., Analysis of the efficiency of a half-toroidal CVT, JSAE Review, Vol. 22, No. 4 (2001), pp. 565-570.

Zhang, Y., Zhang, X. and Tobler, W., A Systematic Model for the Analysis of Contact, Side Slip and Traction of Toroidal Drives, Journal of Mechanical Design, Vol. 122, No. 4 (1999), pp. 523-528. 